\title{
Strong Local Nondeterminism of Spherical Fractional Brownian Motion
}

\author{
Xiaohong Lan * \\ School of Mathematical Sciences \\ University of Science and Technology of China \\ Yimin Xiao ${ }^{\dagger}$ \\ Department of Statistics and Probability \\ Michigan State University
}

September 18, 2018

\begin{abstract}
Let $B=\left\{B(x), x \in \mathbb{S}^{2}\right\}$ be the fractional Brownian motion indexed by the unit sphere $\mathbb{S}^{2}$ with index $0<H \leq \frac{1}{2}$, introduced by Istas [12]. We establish optimal upper and lower bounds for its angular power spectrum $\left\{d_{\ell}, \ell=0,1,2, \ldots\right\}$, and then exploit its high-frequency behavior to establish the property of its strong local nondeterminism of $B$.
\end{abstract}

KEY WORDS: Angular power spectrum, Karhunen-Loève expansion, Spherical fractional Brownian motion, Strong local nondeterminism.

2010 Mathematics Subject Classification: 60G60, 60G22, 60G17.

\section{Introduction}

The spherical fractional Brownian motion (SFBM, for brevity) was introduced by Istas in 2005 [12, as an extension of the spherical Brownian motion of Lévy [18] as well as a spherical analogue of fractional Brownian motion indexed by the Euclidean spaces. Later Istas [13, 14] established the Karhunen-Loève expansion and studied quadratic variations of the spherical fractional Brownian motion.

The purpose of this paper is to investigate the property of strong local nondeterminism (SLND) for the spherical fractional Brownian motion. This is motivated by studies of sample path properties of Gaussian random fields indexed by the Euclidean space $\mathbb{R}^{N}$ and by the currently increasing interest in stochastic

*Corresponding author. Research of X. Lan is supported by NSFC grants 11501538. Email: xhlan@ustc.edu.cn

${ }^{\dagger}$ Research of Y. Xiao is partially supported by NSF grants DMS-1612885 and DMS1607089. E-mail: xiao@stt.msu.edu 
modeling of spherical data in statistics, cosmology and other applied areas (see below).

The concept of local nondeterminism (LND) of a Gaussian process was first introduced by Berman 3 to unify and extend his methods for studying the existence and joint continuity of local times of real-valued Gaussian processes. Roughly speaking, a Gaussian process is said to have the LND property if has locally approximately independent increments, see [3, Lemma 2.3] for precise description. This property allowed Berman to overcome some difficulties caused by the complex dependence structure of a non-Markovian Gaussian process for studying its local times. Pitt 24] and Cuzick [6] extended Berman's LND to Gaussian random fields. However, the property of LND is not enough for establishing fine regularity properties such as the law of the iterated logarithm and the uniform modulus of continuity for the local times or self-intersection local times of Gaussian random fields. For studying these and many other problems on Gaussian random fields, the appropriate properties of strong local nondeterminism (SLND) have proven to be more powerful. Instead of recalling definitions of various forms of strong local nondeterminism for (isotropic or anisotropic) Gaussian random fields indexed by $\mathbb{R}^{N}$ and their applications, we refer to Xiao [26, 27, 28] for more information.

Recently, Lan, Marinucci and Xiao [16] have studied the SLND property of a class of Gaussian random fields indexed by the unit sphere $\mathbb{S}^{2}$, which are also called spherical Gaussian random fields. The main difference between [16] and the aforementioned work for Gaussian fields indexed by the Euclidean space is that [16] takes the spherical geometry of $\mathbb{S}^{2}$ into full consideration and its method relies on harmonic analysis on the sphere. More specifically, Lan, Marinucci and Xiao have considered a centered isotropic Gaussian random field $T=\{T(x), x \in$ $\left.\mathbb{S}^{2}\right\}$. That is, $T$ satisfies

$$
\mathbb{E}(T(x) T(y))=\mathbb{E}(T(g x) T(g y))
$$

for all $g \in S O(3)$ which is the group of rotations in $\mathbb{R}^{3}$. See 20 for a systematic account on random fields on $\mathbb{S}^{2}$. By applying harmonic analytic tools on the sphere, Lan, Marinucci and Xiao [16] have proved that the SLND property of an isotropic Gaussian field $T$ on $\mathbb{S}^{2}$ is determined by the high-frequency behavior of its angular power spectrum. Moreover, by applying SLND, they have established exact uniform modulus of continuity for a class of isotropic Gaussian fields on $\mathbb{S}^{2}$.

Since SFBM $B=\left\{B(x), x \in \mathbb{S}^{2}\right\}$ is not isotropic in the sense of (1), the results on SLND in [16] are not directly applicable. In our approach we will make use of the Karhunen-Loève expansion of the spherical fractional Brownian motion obtained by Istas [13] and derive optimal upper and lower bounds for the coefficients $\left\{d_{\ell}, \ell=0,1, \ldots\right\}$ (see (7) below for the definition). These bounds for $\left\{d_{\ell}\right\}$ correct the last part of Theorem 1 in $[13$ and will be useful for studying the dependence structures and sample path properties of SFBM. This paper provides an important step towards this direction. More specifically, we demonstrate that the coefficients $\left\{d_{\ell}\right\}$ play the same role as the angular power 
spectrum of an isotropic Gaussian field on $\mathbb{S}^{2}$ in [16] and their high frequency behavior determines the property of strong local nondeterminism of SFBM. For this reason, we will also call the sequence $\left\{d_{\ell}, \ell=0,1, \ldots\right\}$ the angular power spectrum of SFBM.

Similarly to the cases of Gaussian random fields indexed by the Euclidean space $\mathbb{R}^{N}$ (cf. [26, 27, 28]), we expect that the SLND property in Theorem 3.2 will be useful for studying regularity (e.g., the exact modulus of continuity, exact modulus of nondifferentiability, etc) and fractal properties of SFBM. This will be carried out in a subsequent paper [17].

Our analysis on SFBM and other spherical Gaussian random fields is strongly motivated by applications in a number of scientific areas, such as geophysics, astrophysics, cosmology, and atmospheric sciences (see e.g. 2, 4, 7, 8, ). Huge data sets from satellite missions such as the Wilkinson Microwave Anisotropy Probe (WMAP) of NASA (see http://map.gsfc.nasa.gov/) and the Planck mission of the European Space Agency (see http://sci.esa.int/planck/53103-planck-cosmology/) have been collected and made publicly available. Spherical random fields (usually assumed to be Gaussian) have been proposed for modeling such data sets.

Related to aforementioned aspects, we also mention that in probability and statistics literature various isotropic or anisotropic Gaussian random fields on $\mathbb{S}^{2}$ have been constructed and studied (see e.g. [9, 10, 11, 15, 23]). Excursion probabilities and topological properties of excursion sets of isotropic Gaussian random fields on $\mathbb{S}^{2}$ have been studied in [5, 21. Many interesting questions on probabilistic and statistical properties of anisotropic Gaussian random fields on the sphere can be raised. In order to study these problems. it would be interesting to establish appropriate properties of strong local nondeterminism for anisotropic Gaussian random fields on $\mathbb{S}^{2}$.

The rest of the paper is organized as follows. In Section 2, we recall briefly some background material on SFBM, including its Karhunen-Loève expansion from [13, and an analysis of its random coefficients. Our main result in this section is Theorem 2.2. which provides optimal upper and lower bounds for the angular power spectrum $\left\{d_{\ell}, \ell=0,1, \ldots\right\}$ of SFBM. In Section 3 , we combine the high frequency behavior of $\left\{d_{\ell}\right\}$ and Proposition 7 in [16 to establish the property of strong local nondeterminism for SFBM.

\section{SFBM and asymptotic behavior of its angular power spectrum}

Let $N$ be the North pole on $\mathbb{S}^{2}$, and $d_{\mathbb{S}^{2}}(x, y)$ the geodesic distance between $x$ and $y$ on $\mathbb{S}^{2}$. Recall from Istas [12] the definition of SFBM.

Definition 2.1 The SFBM $B=\left\{B(x), x \in \mathbb{S}^{2}\right\}$ is a centered Gaussian random field with

$$
B(N)=0, \text { a.s. }
$$

and

$$
\mathbb{E}[B(x)-B(y)]^{2}=d_{\mathbb{S}^{2}}(x, y)^{2 H},
$$


for any $x, y \in \mathbb{S}^{2}$ and $0<H \leq \frac{1}{2}$.

It is well known that fractional Brownian motion indexed by $\mathbb{R}^{d}$ can be defined for every $0<H \leq 1$. This is different from the case when the index set is $\mathbb{S}^{2}$. Istas [12 proved that SFBM exists if and only if the Hurst index $H \in\left(0, \frac{1}{2}\right]$. When $H=\frac{1}{2}$, it is the classical Lévy spherical Brownian motion, see [18, 22].

Our work is based on the following Karhunen-Loève expansion of $\{B(x)$, $\left.x \in \mathbb{S}^{2}\right\}$ proved by Istas [13, Theorem 1]:

$$
B(x)=\sum_{\ell=0}^{\infty} \sum_{m=-\ell}^{\ell} i \sqrt{\pi d_{\ell}} \varepsilon_{\ell m}\left(Y_{\ell m}(x)-Y_{\ell m}(N)\right) .
$$

Here $\left\{Y_{\ell m}: \ell=0,1, \ldots, m=-\ell, \ldots, \ell\right\}$ are the spherical harmonic functions on $\mathbb{S}^{2}$; that is, they are eigenfunctions of the spherical Laplacian:

$$
\Delta_{\mathbb{S}^{2}}=\frac{1}{\sin \theta} \frac{\partial}{\partial \theta}\left\{\sin \theta \frac{\partial}{\partial \theta}\right\}+\frac{1}{\sin ^{2} \theta} \frac{\partial^{2}}{\partial \phi^{2}},
$$

where $(\theta, \phi) \in[0, \pi] \times[0,2 \pi)$ denotes the spherical coordinates of $x \in \mathbb{S}^{2}$. More precisely, $\left\{Y_{\ell m}: \ell=0,1, \ldots, m=-\ell, \ldots, \ell\right\}$ satisfy

$$
\Delta_{\mathbb{S}^{2}} Y_{\ell m}(\theta, \phi)=-\ell(\ell+1) Y_{\ell m}(\theta, \phi) .
$$

It is known (cf. 20]) that $\left\{Y_{\ell m}: \ell=0,1, \ldots, m=-\ell, \ldots, \ell\right\}$ form an orthonormal basis for the space $L^{2}\left(\mathbb{S}^{2}, d \sigma\right)$, where $d \sigma=\sin \theta d \theta d \phi$ is the Lebesgue measure on $\mathbb{S}^{2}$. An explicit form for spherical harmonics is given by

$$
\begin{array}{ll}
Y_{\ell m}(\theta, \phi)=\sqrt{\frac{2 \ell+1}{4 \pi} \frac{(\ell-m) !}{(\ell+m) !}} P_{\ell m}(\cos \theta) e^{i m \phi}, & \text { for } m \geq 0, \\
Y_{\ell m}(\theta, \phi)=(-1)^{m} \bar{Y}_{\ell,-m}(\theta, \phi), & \text { for } m<0,
\end{array}
$$

where $\bar{z}$ denotes the complex conjugate of $z \in \mathbb{C}$, and $P_{\ell m}(\cos \vartheta)$ are the associated Legendre functions (cf. [20, pp.315-316]) defined in terms of the Legendre polynomials $\left\{P_{\ell}, \ell=0,1, \ldots\right\}$ as

$$
P_{\ell m}(x)=(-1)^{m}\left(1-x^{2}\right)^{m / 2} \frac{d^{m}}{d x^{m}} P_{\ell}(x)
$$

for $m=0,1, \ldots, \ell$, and

$$
P_{\ell m}(x)=(-1)^{m} \frac{(\ell-m) !}{(\ell+m) !} P_{\ell,-m}(x)
$$

for $m$ negative. Moreover, the following orthonormality property holds:

$$
\int_{\mathbb{S}^{2}} Y_{l m}(x) \bar{Y}_{l^{\prime} m^{\prime}}(x) d \sigma(x)=\delta_{l}^{l^{\prime}} \delta_{m}^{m^{\prime}}
$$


Now recall from [13] that, for all $\ell \geq 0$, the coefficients $d_{\ell}$ in (44) are defined by

$$
d_{\ell}=\frac{1}{2 \pi} \int_{\mathbb{S}^{2}} d_{\mathbb{S}^{2}}(x, N)^{2 H} P_{\ell}(\langle x, N\rangle) d \sigma(x),
$$

where $\langle\cdot, \cdot\rangle$ is the usual inner product in $\mathbb{R}^{3}$. Similarly to the angular power spectrum of an isotropic Gaussian random field on $\mathbb{S}^{2}$, the sequence $\left\{d_{\ell}, \ell=0,1,2, \ldots\right\}$ plays an important role in determining the dependence structure and other probabilistic properties of the SFBM $\left\{B(x), x \in \mathbb{S}^{2}\right\}$. Hence we will also refer to $\left\{d_{\ell}, \ell=0,1,2, \ldots\right\}$ as the angular power spectrum of SFBM.

Moreover, it follows from Theorem 1 of Istas [13] that the Karhunen-Loève expansion (4) holds in $L^{2}\left(\mathbb{S}^{2} \times \Omega, d \sigma \otimes \mathbb{P}\right)$ sense and in $L^{2}(\mathbb{P})$ sense for every fixed $x \in \mathbb{S}^{2}$. Hence one can represent the random coefficients $\varepsilon_{\ell m}, \ell=$ $0,1, \ldots, m=-\ell, \ldots, \ell$ in (4) by

$$
\varepsilon_{\ell m}=-i\left(\pi d_{\ell}\right)^{-1 / 2} \int_{\mathbb{S}^{2}} B(x) \overline{Y_{\ell m}}(x) d \sigma(x),
$$

where the equality holds in $L^{2}(\mathbb{P})$ sense. Notice that $\left\{\varepsilon_{\ell m}\right\}_{l m}$ is a set of complexvalued Gaussian random variables. Obviously, $\mathbb{E}\left(\varepsilon_{\ell m}\right)=0$ for all $\ell, m$, due to the zero-mean property of $B(x)$. Moreover, recall (5) and (6), we can verify that

$$
\varepsilon_{\ell m}=(-i)^{m} \varepsilon_{\ell,-m} \text { and } \mathbb{E}\left(\varepsilon_{\ell_{1} m_{2}} \bar{\varepsilon}_{\ell_{2} m_{2}}\right)=\delta_{\ell_{2}}^{\ell_{1}} \delta_{m_{2}}^{m_{1}} .
$$

Therefore, $\left\{\varepsilon_{\ell m}, \ell \geq 0, m=0,1, \ldots, \ell\right\}$ is a set of i.i.d. standard complex Gaussian random variables.

The following is the main result of this section. The bounds in (9) correct the last part of Theorem 1 in [13. The high-frequency behavior of $d_{\ell}$ in (9) is essential for proving the SLND property of $B$ in Theorem 3.2 below. Together, they will allow us to study precise analytic and geometric properties of the sample functions of SFBM. See [17] for further information.

Theorem 2.2 Let $\left\{B(x), x \in \mathbb{S}^{2}\right\}$ be the spherical fractional Brownian motion of index $H \in(0,1 / 2]$. There exists a uniform constant $K_{1}$ such that

$$
\begin{gathered}
K_{1}^{-1} \leq d_{0} \leq K_{1} \\
K_{1}^{-1} \ell^{-(2 H+2)} \leq d_{\ell} \leq K_{1} \ell^{-(2 H+2)} \quad \text { for all } \ell=1,2, \ldots
\end{gathered}
$$

Proof. We work in spherical coordinates $(\theta, \phi)$ with $\theta \in[0, \pi]$ and $\phi \in[0,2 \pi]$. By the definition of $d_{\ell}$ in (7) and a change of variable $x=\cos \theta$ we obtain

$$
d_{\ell}=\int_{0}^{\pi} \theta^{2 H} P_{\ell}(\cos \theta) \sin \theta d \theta .
$$

Recall from [13] the Dirichlet-Mehler representation for $P_{\ell}(\cos \theta)(0<\theta<\pi)$,

$$
P_{\ell}(\cos \theta)=\frac{\sqrt{2}}{\pi} \int_{\theta}^{\pi} \frac{\sin \left(\left(\ell+\frac{1}{2}\right) \varphi\right)}{\sqrt{\cos \theta-\cos \varphi}} d \varphi
$$


we see that (10) becomes

$$
\begin{aligned}
d_{\ell} & =\frac{\sqrt{2}}{\pi} \int_{0}^{\pi} \theta^{2 H} \sin \theta \int_{\theta}^{\pi} \frac{\sin \left(\left(\ell+\frac{1}{2}\right) \varphi\right)}{\sqrt{\cos \theta-\cos \varphi}} d \varphi d \theta \\
& =\frac{\sqrt{2}}{\pi} \int_{0}^{\pi} \sin \left(\left(\ell+\frac{1}{2}\right) \varphi\right)\left[\int_{0}^{\varphi} \frac{\theta^{2 H} \sin \theta}{\sqrt{\cos \theta-\cos \varphi}} d \theta\right] d \varphi
\end{aligned}
$$

From the elementary fact that $\sin \frac{\theta}{2} \leq \frac{\theta}{2} \leq \theta\left[1-\frac{1}{6}\left(\frac{\theta}{2}\right)^{2}\right] \leq 2 \sin \frac{\theta}{2}$ for all $0 \leq \theta \leq \pi$, it follow that

$$
\left(\sin \frac{\theta}{2}\right)^{2 H} \leq\left(\frac{\theta}{2}\right)^{2 H} \leq 2^{2 H} \cdot\left(\sin \frac{\theta}{2}\right)^{2 H}, \quad 0 \leq \theta \leq \pi .
$$

Hence, we have

$$
\widetilde{d}_{\ell} \leq d_{\ell} \leq 2^{2 H} \widetilde{d}_{\ell}
$$

where

$$
\widetilde{d}_{\ell}=\frac{2^{2 H+1 / 2}}{\pi} \int_{0}^{\pi} \sin \left(\left(\ell+\frac{1}{2}\right) \varphi\right) \int_{0}^{\varphi} \frac{\left(\sin \frac{\theta}{2}\right)^{2 H} \sin \theta}{\sqrt{\cos \theta-\cos \varphi}} d \theta d \varphi .
$$

Meanwhile, by a change of variable $u=\sin \frac{\theta}{2}$ in the inside integral, we have

$$
\begin{aligned}
\int_{0}^{\varphi} \frac{\left(\sin \frac{\theta}{2}\right)^{2 H} \sin \theta}{\sqrt{\cos \theta-\cos \varphi}} d \theta & =2^{\frac{3}{2}} \int_{0}^{\sin \frac{\varphi}{2}} \frac{u^{2 H+1}}{\sqrt{\sin ^{2} \frac{\varphi}{2}-u^{2}}} d u \\
& =2^{\frac{1}{2}}\left(\sin \frac{\varphi}{2}\right)^{2 H+1} \int_{0}^{1} \frac{v^{H}}{\sqrt{1-v}} d v \\
& =2^{\frac{1}{2}} B\left(H+1, \frac{1}{2}\right)\left(\sin \frac{\varphi}{2}\right)^{2 H+1}
\end{aligned}
$$

where $B(\cdot, \cdot)$ is the Beta function defined on $\mathbb{R}^{+} \times \mathbb{R}^{+}$. Consequently,

$$
\widetilde{d}_{\ell}=\frac{2^{2 H+1}}{\pi} B\left(H+1, \frac{1}{2}\right) \int_{0}^{\pi} \sin \left(\left(\ell+\frac{1}{2}\right) \varphi\right)\left(\sin \frac{\varphi}{2}\right)^{2 H+1} d \varphi .
$$

For $\ell=0$,

$$
\widetilde{d}_{0}=\frac{2^{2 H+1}}{\pi} B\left(H+1, \frac{1}{2}\right) \int_{0}^{\pi}\left(\sin \frac{\varphi}{2}\right)^{2 H+2} d \varphi
$$

which is readily seen that $\left|\widetilde{d}_{0}\right| \leq 8$. For $\ell \geq 1$, we will make use of some techniques from complex analysis. Let $z=e^{i \varphi / 2}$, then the integral in (12) can be written as

$$
\int_{0}^{\pi} \sin \left(\left(\ell+\frac{1}{2}\right) \varphi\right)\left(\sin \frac{\varphi}{2}\right)^{2 H+1} d \varphi=2 \operatorname{Im} \int_{\mathcal{C}} f_{\ell}(z) d z
$$


where $\mathcal{C}=\left\{z: z=e^{i t}, 0 \leq t \leq \pi / 2\right\}$ is the unit circle in the first quadrant with the direction counterclockwise and $f_{\ell}(z)$ is the principal branch of the complex function

$$
F_{\ell}(z)=\frac{z^{2 \ell}}{2^{2 H} i^{2 H+1}}\left(z-\frac{1}{z}\right)^{2 H+1}, \quad z \in \mathbb{C},
$$

Obviously, $f_{\ell}(z)$ is analytic in $\mathbb{C} \backslash\{0, \pm 1\}$. Moreover, let $\epsilon>0$ be an arbitary small value, $\mathcal{L}_{1}$ and $\mathcal{L}_{2}$ the two line segments defined by

$$
\mathcal{L}_{1}=\{z: z=i(1-t), \epsilon \leq t \leq 1\}
$$

and

$$
\mathcal{L}_{2}=\{z: z=t, \epsilon \leq t \leq 1-\epsilon\} ;
$$

Meantime, let $\mathcal{C}_{1}$ and $\mathcal{C}_{2}$ be the circles with radius $\epsilon$ in the first and second quadrants, defined respectively by

$$
\mathcal{C}_{1}=\left\{z: z=\epsilon e^{i t}, 0 \leq t \leq \pi / 2\right\}
$$

and

$$
\mathcal{C}_{2}=\left\{z: z=\epsilon e^{i(\pi-t)}+1,0 \leq t \leq \pi / 2\right\} .
$$

Now consider the following integrals

$$
\begin{gathered}
\int_{\mathcal{L}_{1}} f_{\ell}(z) d z=\frac{i^{2 \ell-2 H-1}}{2^{2 H} i^{2 H+1}} \int_{\epsilon}^{1}(1-t)^{2 \ell-2 H-1}\left[-(1-t)^{2}-1\right]^{2 H+1} d t \\
=\frac{(-1)^{\ell}}{2^{2 H}} \int_{\epsilon}^{1} u^{2 \ell-2 H-1}\left[u^{2}+1\right]^{2 H+1} d t \\
\int_{\mathcal{L}_{2}} f_{\ell}(z) d z=\frac{1}{2^{2 H} i^{2 H+1}} \int_{\epsilon}^{1} t^{2 \ell-2 H-1}\left(t^{2}-1\right)^{2 H+1} d t \\
=\frac{i^{2 H+1}}{2^{2 H}} \int_{\epsilon}^{1}(1-v)^{\ell-H-1 / 2} v^{2 H+1} d t
\end{gathered}
$$

and

$$
\begin{gathered}
\int_{\mathcal{C}_{1}} f_{\ell}(z) d z=\frac{1}{2^{2 H} i^{2 H+1}} \int_{0}^{\frac{\pi}{2}}\left(\epsilon e^{i(\pi-t)}\right)^{2 \ell-2 H-1}\left(\epsilon^{2} e^{2 i(\pi-t)}-1\right)^{2 H+1} d t \\
\int_{\mathcal{C}_{2}} f_{\ell}(z) d z=\frac{1}{2^{2 H} i^{2 H+1}} \int_{0}^{\frac{\pi}{2}}\left(1+\epsilon e^{i(\pi-t)}\right)^{2 \ell-2 H-1}\left(\epsilon^{2} e^{2 i(\pi-t)}+2 \epsilon e^{i(\pi-t)}\right)^{2 H+1} d t .
\end{gathered}
$$

Careful calculations show that

$$
\operatorname{Im} \int_{\mathcal{L}_{1}} f_{\ell}(z) d z=0, \lim _{\epsilon \rightarrow 0}\left|\int_{\mathcal{C}_{j}} f_{\ell}(z) d z\right|=0, j=1,2
$$


and

$$
\lim _{\epsilon \rightarrow 0} \int_{\mathcal{L}_{2}} f_{\ell}(z) d z=\frac{i^{2 H+1}}{2^{2 H}} B(2 H+2, \ell-H+1 / 2)
$$

Thus, by the Cauchy integral theorem in complex analysis, we have

$$
\begin{aligned}
\int_{\mathcal{C}} f_{\ell}(z) d z & =-\lim _{\epsilon \rightarrow 0} \int_{\mathcal{L}_{1}+\mathcal{C}_{1}+\mathcal{L}_{2}+\mathcal{C}_{2}} f_{\ell}(z) d z \\
& =-\lim _{\epsilon \rightarrow 0} \int_{\mathcal{L}_{1}} f_{\ell}(z) d z-\frac{i^{2 H+1}}{2^{2 H}} B\left(2 H+2, \ell-H+\frac{1}{2}\right)
\end{aligned}
$$

which leads to that

$$
\operatorname{Im} \int_{\mathcal{C}} f_{\ell}(z) d z=2^{-2 H-1} B\left(2 H+2, \ell-H+\frac{1}{2}\right) \sin \left[\left(H+\frac{1}{2}\right) \pi\right] .
$$

in view of the equalities (14) and (15). Therefore, by combining the equalities (16) and (12), (13) above, we obtain that

$$
\widetilde{d}_{\ell}=\frac{2}{\pi} B\left(H+1, \frac{1}{2}\right) B\left(2 H+2, \ell-H+\frac{1}{2}\right) \sin \left[\left(H+\frac{1}{2}\right) \pi\right] .
$$

Here, recall the formula

$$
B(a, b)=\frac{\Gamma(a) \Gamma(b)}{\Gamma(a+b)}, a>0, b>0
$$

with Gamma function $\Gamma(y)=(y-1) \Gamma(y-1)$ for any $y>1$ and the stirling's approximation

$$
\Gamma(y)=\sqrt{2 \pi y}\left(\frac{y}{e}\right)^{y}\left(1+O\left(\frac{1}{y}\right)\right), \quad \text { as } y \rightarrow \infty,
$$

where the error term $O\left(\frac{1}{y}\right)$ being the same order as $\frac{1}{y}$, we derive the following estimates: there exists a uniform constant $C_{1}$ such that for any $\ell \geq 1$,

$$
C_{1}^{-1} \ell^{-(2 H+2)} \leq \tilde{d}_{\ell} \leq C_{1} \ell^{-(2 H+2)}
$$

Let $K_{1}=2 C_{1}$, then the inequalities in (9) are derived in view of (11).

\section{Strong local nondeterminism}

In order to prove the strong local nondeterminism property of $\left\{B(x), x \in \mathbb{S}^{2}\right\}$, we first recall the following lemma, which is a consequence of Proposition 7 in [16]. 
Lemma 3.1 Assume a sequence $\left\{d_{\ell}, \ell=0,1, \ldots\right\}$ satisfies the condition (9). Then there exists a constant $C_{2}>0$ depending on $H$ only, such that for all choices of $n \in \mathbb{N}$, all $x, x_{1}, \ldots, x_{n} \in \mathbb{S}^{2}$, and $\gamma_{j} \in \mathbb{R}, j=1,2, \ldots, n$, we have

$$
\sum_{\ell=0}^{\infty} \sum_{m=-\ell}^{\ell} d_{\ell}\left[Y_{\ell m}(x)-\sum_{j=1}^{n} \gamma_{j} Y_{\ell m}\left(x_{j}\right)\right]^{2} \geq C_{2} \varepsilon^{2 H}
$$

where $\varepsilon=\min \left\{d_{\mathbb{S}^{2}}\left(x, x_{k}\right), k=1, \ldots, n\right\}$.

Now we are ready to state and prove the following theorem. Its conclusion is referred to as the property of strong local nondeterminism of SFBM $\left\{B(x), x \in \mathbb{S}^{2}\right\}$.

Theorem 3.2 For a $S F B M\left\{B(x), x \in \mathbb{S}^{2}\right\}$, there exists a constant $K_{2}>0$ depending only on the Hurst index $H \in(0,1 / 2]$, such that for all integers $n \geq 1$ and all $x, x_{1}, \ldots, x_{n} \in \mathbb{S}^{2}$, we have

$$
\operatorname{Var}\left(B(x) \mid B\left(x_{1}\right), \ldots, B\left(x_{n}\right)\right) \geq K_{2} \min _{0 \leq k \leq n} d_{\mathbb{S}^{2}}\left(x, x_{k}\right)^{2 H},
$$

where $\operatorname{Var}\left(B(x) \mid B\left(x_{1}\right), \ldots, B\left(x_{n}\right)\right)$ denotes the conditional variance of $B(x)$ given $B\left(x_{1}\right), \ldots, B\left(x_{n}\right)$, and $x_{0}=N$.

Remark 3.3 Note that the strong local nondeterminism (18) here is slightly different from the SLND property proved in [16] for isotropic Gaussian random fields: the minimum on the right hand side of (18) is not only taken over $x_{1}, \ldots, x_{n}$ but also over $x_{0}=N$. This is because of the assumption $B(N)=0$ in the definition of SFBM. From statistics viewpoint, $\operatorname{Var}\left(B(x) \mid B\left(x_{1}\right), \ldots, B\left(x_{n}\right)\right)$ is the squared error of predicting the value $B(x)$, given observations of $B$ at locations $x_{1}, \ldots, x_{n}$. Since we already know that $B(N)=0$, this information may reduce the prediction error.

Proof of Theorem 3.2. It is known that for a Gaussian random field $B$,

$$
\operatorname{Var}\left(B(x) \mid B\left(x_{1}\right), \ldots, B\left(x_{n}\right)\right)=\inf \left\{\mathbb{E}\left[\left(B(x)-\sum_{j=1}^{n} \gamma_{j} B\left(x_{j}\right)\right)^{2}\right]\right\},
$$

where the infimum is taken over all $\left(\gamma_{1}, \ldots, \gamma_{n}\right) \in \mathbb{R}^{n}$. Hence, in order to establish (18), it is sufficient to prove that there exists a positive constant $C_{3}$ such that

$$
\mathbb{E}\left[\left(B(x)-\sum_{j=1}^{n} \gamma_{j} B\left(x_{j}\right)\right)^{2}\right] \geq C_{3} \varepsilon^{2 H}
$$

holds for all $\gamma_{1}, \ldots, \gamma_{n} \in \mathbb{R}$, where $\varepsilon=\min \left\{d_{\mathbb{S}^{2}}\left(x, x_{k}\right), k=0, \ldots, n\right\}$. Let $\gamma_{0}=$ $1-\sum_{j=1}^{n} \gamma_{j}$, then it follows from the Karhunen-Loève expansion (44) of $B(x)$ and 
the properties of random coefficients $\left\{\varepsilon_{\ell m}\right\}_{l m}$ in (8) that the left hand side of (19) is equal to

$$
\begin{aligned}
& \mathbb{E}\left\{\left[\sum_{\ell=0}^{\infty} \sum_{m=-\ell}^{\ell} i \sqrt{\pi d_{\ell}} \varepsilon_{\ell m}\left(Y_{\ell m}(x)-\sum_{j=0}^{n} \gamma_{j} Y_{\ell m}\left(x_{j}\right)\right)\right]^{2}\right\} \\
& =\sum_{\ell=0}^{\infty} \sum_{m=-\ell}^{\ell} \pi d_{\ell} \mathbb{E}\left(\left|\varepsilon_{\ell m}\right|^{2}\right)\left|Y_{\ell m}(x)-\sum_{j=0}^{n} \gamma_{j} Y_{\ell m}\left(x_{j}\right)\right|^{2} \\
& =\pi \sum_{\ell=0}^{\infty} \sum_{m=-\ell}^{\ell} d_{\ell}\left|Y_{\ell m}(x)-\sum_{j=0}^{n} \gamma_{j} Y_{\ell m}\left(x_{j}\right)\right|^{2}
\end{aligned}
$$

where $x_{0}=N$. Therefore (19) is an immediate consequence of Lemma 3.1 in view of the equalities (20). This completes the proof of (18).

\section{References}

[1] Adler, R. J. and Taylor, J. E. (2007). Random Fields and Geometry. Springer, New York.

[2] Alkhaled, A. A., Michalak, A. M., Kawa, S. R., Olsen, S. C. and Wang, J.W. (2008). A global evaluation of the regional spatial variability of column integrated CO2 distributions. J Geophys Res 113: D20303.

[3] Berman, S. M. (1973), Local nondeterminism and local times of Gaussian processes. Indiana Univ. Math. J. 23, 69-94.

[4] Cabella, P. and Marinucci, D. (2009). Statistical challenges in the analysis of Cosmic Microwave Background radiation. Ann. Appl. Statist. 2, 61-95.

[5] Cheng, D. and Xiao, Y. (2016). Excursion probability of Gaussian random fields on sphere. Bernoulli 22, 1113-1130.

[6] Cuzick, J. (1982), Multiple points of a Gaussian vector field. Z. Wahrsch. Verw. Gebiete 61, 431-436.

[7] de Boyer Montégut, C., Madec, G., Fischer, A. S., Lazar, A. and Iudicone, D. (2004). Mixed layer depth over the global ocean: an examination of profile data and a profile-based climatology. J. Geophys. Res. 109, C12003.

[8] Dodelson, S. (2003). Modern Cosmology. Academic Press.

[9] Estrade, A. and Istas, J. (2010). Ball throwing on spheres. Bernoulli 16, 953-970.

[10] Huang, C., Zhang, H. and Robeson S. M. (2011). On the validity of commonly used covariance and variogram functions on the sphere. Math. Geosci. 43, 721-733. 
[11] Huang, C., Zhang, H. and Robeson S. M. (2012). A simplified representation of the covariance structure of axially symmetric processes on the sphere. Stat. Prob. Lett. 82, 1346-1351.

[12] Istas, J. (2005). Spherical and hyperbolic fractional Brownian motion. Elect. Comm. Probab. 10, 254-262.

[13] Istas, J. (2006). Karhunen-Loève expansion of spherical fractional Brownian motions. Statist. Probab. Lett. 76, 1578-1583.

[14] Istas, J. (2007). Quadratic variations of spherical fractional Brownian motions. Stoch. Process. Appl. 117, 476-486.

[15] Jun, M. and Stein, M. L. (2008). Nonstationary covariance models for global data. Ann. Appl. Statist. 2, 1271-1289.

[16] Lan, X., Marinucci, D. and Xiao, Y. (2016). Strong local nondeterminism and exact modulus of continuity for spherical Gaussian fields, Stoch. Process. Appl., accepted, arXiv:1603.03642.

[17] Lan, X. and Xiao, Y. (2017). Exact moduli of continuity and nonwhere differentiability of spherical fractional Brownian motion. In Preparation.

[18] Lévy, P. (1965). Processus Stochastiques et Mouvement Brownien, Gauthier-Villars.

[19] Marcus, M. B. and Rosen J. (2006). Markov Processes, Gaussian Processes, and Local Times, Cambridge University Press, Cambridge.

[20] Marinucci, D. and Peccati, G. (2011). Random Fields on the Sphere. Representation, Limit Theorem and Cosmological Applications, Cambridge University Press, Cambridge.

[21] Marinucci, D. and Vadlamani, S. (2016). High-frequency asymptotics for Lipschitz-Killing curvatures of excursion sets on the sphere. Ann. Appl. Probab. 26, 462-506.

[22] Noda, A. (1987). Generalized Radon transform and Lévy's Brownian motion. Nagoya Math. J. 105, 71-87.

[23] Panchenko, D. and Talagrand, M. (2007). On the overlap in the multiple spherical SK models. Ann. Probab. 35, 2321-2355.

[24] Pitt, L. D. (1978), Local times for Gaussian vector fields. Indiana Univ. Math. J. 27, 309-330.

[25] Stein, E. M. and Weiss, G. (1971). Introduction to Fourier Analysis on Euclidean Spaces. Princeton University Press. 
[26] Xiao, Y. (2007). Strong local nondeterminism and sample path properties of Gaussian random fields. Asymptotic Theory in Probability and Statistics with Applications (Tze Leung Lai, Qiman Shao, Lianfen Qian, editors), pp. 136-176, Higher Education Press, Beijing.

[27] Xiao, Y. (2009). Sample path properties of anisotropic Gaussian random fields. In: A Minicourse on Stochastic Partial Differential Equations, (D. Khoshnevisan and F. Rassoul-Agha, editors), Lecture Notes in Math. 1962, pp. 145-212. Springer, New York.

[28] Xiao, Y. (2013). Recent developments on fractal properties of Gaussian random fields. In: Further Developments in Fractals and Related Fields, (J. Barral and S. Seuret, eds.) pp.255-288, Springer, New York. 